\title{
Tipología de Atributos Ponderada en Base a Normas de Producción de Atributos Semánticos
}

\author{
Mauro MacIntyre \\ Instituto de Psicología Básica, Aplicada y Tecnología, Universidad Nacional de Mar \\ del Plata, CONICET, Facultad de Psicología, Mar del Plata, Argentina \\ Leticia Vivas ${ }^{1}$ \\ Instituto de Psicología Básica, Aplicada y Tecnología, Universidad Nacional de Mar \\ del Plata, CONICET, Facultad de Psicología, Mar del Plata, Argentina \\ CONICET, Argentina \\ Jorge Vivas \\ Instituto de Psicología Básica, Aplicada y Tecnología, Universidad Nacional de Mar \\ del Plata, CONICET, Facultad de Psicología, Mar del Plata, Argentina
}

\section{Resumen}

La tipología de atributos propuestas por las teorías sensorio-funcionales para explicar las diferencias entre dominios (seres vivos y objetos inanimados) cuenta con diversas críticas: (a) imprecisa definición del tipo de atributo; (b) limitaciones de la taxonomía dicotómica; (c) insuficiencia de la variable frecuencia de producción para discriminar entre dominios. El presente trabajo pretende colaborar a la superación de estas dificultades proponiendo un análisis basado en una taxonomía compleja y partiendo de datos empíricos obtenidos a partir de Normas de Producción de Atributos Semánticos en Español, considerando la frecuencia y el orden de producción de los atributos. Se trabajó sobre una muestra de 180 adultos sanos hispanoparlantes que emitieron atributos definidores para 90 conceptos concretos. Los resultados refleja similitudes entre los dominios sobre la primacía de atributos supraordinados y diferencias en cuanto al peso de los atributos dinámicos (funcionales y comportamentales) y sensoriales. Esta información contribuye a la caracterización de la estructura composicional de los atributos que conforman la representación semántica de los conceptos concretos.

Palabras clave: Atributos semánticos, normas de producción de atributos semánticos, dominios semánticos, accesibilidad.

\section{Tipologia de Atributos Ponderada Baseadas em Normas de Produção Atributos Semânticos}

\section{Resumo}

A tipologia de atributos propostas pelas teorias sensório-funcionais para explicar as diferenças entre domínios (seres vivos e objetos inanimados) tem críticas mistas: (a) definição imprecisa do tipo de atributo; (b) limitações da taxonomia dicotômica; (c) insuficiência da variável frequência de produção para discriminar entre domínios. Este trabalho pretende contribuir para superar essas dificuldades, propondo uma taxonomia mais complexa e partindo de dados empíricos obtidos de Normas de Produção de Atributos Semânticos em Espanhol, considerando a freqüência e ordem de produção dos atributos.

Dirección postal: Deán Funes 3350, B7602AYL, Mar del Plata, Buenos Aires, Argentina. E-mail: 1vivas@ mdp.edu.ar

Agências de fomento: Universidad Nacional de Mar del Plata. 
O trabalho foi feito em uma amostra de 180 adultos saudáveis falantes de espanhol que emitiram atributos definidores para 90 conceitos concretos. Os resultados refletem semelhanças entre domínios na primazia de atributos superordenados e diferenças quanto ao peso dos atributos dinâmicos (funcionais e comportamentais) e sensoriais. Esta informação contribui para a caracterização da composição estuctural dos atributos que fazem a representação semântica de conceitos específicos.

Palavras-chave: Atributos semânticos, normas de produção de atributos semânticos, domínios semânticos, acessibilidade.

\title{
Weighted Feature Typology Based on Semantic Feature Production Norms
}

\begin{abstract}
Feature taxonomy proposed by sensory-functional theories to differentiate between domains (living or non-living things) received a number of critics: (a) the vagueness of the definition of attribute types; (b) the limitations of the dichotomous taxonomy; (c) the insufficiency of feature production variable to discriminate between domains. This paper aims to contribute to overcome these difficulties by proposing an analysis based on a complex taxonomy supported on empirical data obtained from Semantic Feature Production Norms in Spanish, considering features' frequency and order of production. Data was collected from a sample of 180 Spanish speaking healthy adults who produced defining features for 90 concrete concepts. The results show similarities between domains, in so far as to the primacy of superordinate attributes, and differences in the weight of the dynamic (functional and behavioral) and sensory features. This information is worthy to characterize the compositional structure of the features that constitute the semantic representation of concrete concepts.
\end{abstract}

Keywords: Semantic feature, semantic feature production norms, semantic domains, accessibility.

El problema sobre los tipos de atributos que predominan en la representación de los dominios semánticos (seres vivos vs objetos inanimados) es un tema planteado inicialmente por los modelos sensorio-funcionales. Estos modelos proponen que el conocimiento conceptual está organizado en función de distintas modalidades sensoriales y que, para cada dominio semántico, predomina un tipo de modalidad (Farah \& McClelland, 1991; Warrington \& Shallice, 1984). De este modo, tradicionalmente se afirma que el dominio de seres vivos se caracteriza por una preponderancia de atributos de tipo sensoriales (por ejemplo, atributos referidos al color o la forma), mientras que el dominio de objetos inanimados o artefactos se caracteriza por atributos funcionales o asociativos (por ejemplo, sirve para cortar o se encuentra en un taller; MartínezCuitiño, 2007). Incluso se han propuesto correlatos neurofisiológicos para cada una de las modalidades (Fargier et al., 2014).
Los modelos sensorio-funcionales han recibido críticas de diversa índole tanto por la disparidad de sus resultados como por la metodología utilizada (Capitani, Laiacona, Mahon, \& Caramazza, 2003; McRae \& Cree, 2002; Peraita, 2006). En lo que respecta a las limitaciones metodológicas para caracterizar a los dominios semánticos las críticas se pueden agrupar en tres grandes categorías: (a) Imprecisión en la definición de los tipos de atributo "sensorial" y "funcional"; (b) Limitaciones de la taxonomía dicotómica; (c) La mera frecuencia de producción de los atributos resulta insuficiente para discriminar entre dominios y se necesita de la consideración de otras cualidades para dar cuenta de estas diferencias.

En lo que sigue se presentan los trabajos que han avanzado en estas tres líneas de críticas y se finaliza con el aporte de la presente investigación a la descripción de los tipos de atributos que caracterizan a los dominios semánticos a partir de 
los datos obtenidos de Normas de Producción de Atributos Semánticos en Español empíricamente derivadas (Vivas, Vivas, Comesaña, García Coni, \& Vorano, 2016).

\section{Imprecisión en la Definición de los Tipos de Atributo "Sensorial" y "Funcional"}

Los criterios utilizados para clasificar a los atributos en sensoriales y funcionales no son los mismos en todos los trabajos que se han enmarcado en esta concepción. Cabe citar un trabajo realizado por McRae y Cree (2002) en el cual se analizan y ponen a prueba las diferencias entre dominios semánticos de acuerdo con las distintas concepciones de los términos "sensorial" y "funcional", dando cuenta de las variaciones que se observan. Estos autores, resaltan que a lo largo de los estudios enmarcados en las teorías sensorio-funcionales los atributos incluidos en los atributos sensoriales han variado desde la inclusión exclusiva de atributos visuales (Farah $\&$ McClelland, 1991) hasta la inclusión de cualquier tipo de atributo sensorial, ya sea textura, olfato, tacto (Caramazza \& Shelton, 1998; Devlin et al., 1998). Por otra parte, en el estudio de Garrard, Lambon Ralph, Hodges y Patterson (2001) se muestra que, si bien existe una diferencia positiva relativamente significativa entre los atributos sensoriales para la categoría seres vivos en comparación con los conceptos de la categoría de objetivos inanimados, las diferencias en los atributos funcionales entre los dos dominios no fueron suficientemente grandes. Esto se debe, según estos autores, al rol asignado a los atributos enciclopédicos dentro de las clasificaciones, ya que algunos autores los ubican dentro de los funcionales y otro en una categoría propia. Del mismo modo, hay autores que consideran a los atributos funcionales dentro de las relaciones temáticas (relaciones de co-ocurrencia de objetos en contextos específicos) incluyendo dentro de esta categoría a relaciones como "tiza-pizarrón" (Kalènine et al., 2009). Consecuentemente observan mayores relaciones de este tipo para los objetos inanimados. Mientras que otros consideran que una relación funcional es aquella vincula al concepto directamente con su función (eg. "pala-sirve para cavar"; Johnson, Hermann, \& Bonilla, 1995).

Está claro que si no se cuenta con una taxonomía de los tipos de atributos que sea lo suficientemente explícita y justificada, las inferencias realizadas sobre las diferencias entre los dominios y categorías semánticas resultan endebles. En este sentido, Wu y Barsalou (2009) han propuesto una taxonomía, basada en datos empíricos, a partir de la producción de los participantes en una tarea de generación de atributos semánticos. Esta taxonomía fue elaborada partiendo del modelo de Conceptualización Situada (Barsalou, 2005) y propone un sistema de clasificación que incluye: Taxonómicos, Propiedades Situacionales, Propiedades de la Entidad, Propiedades Introspectivas y Misceláneas. Cada una de estas clases está constituida por numerosos subtipos. Los factores tomados en cuenta por los autores para su elaboración fueron los siguientes: (a) cubrir la extensa producción de los sujetos en tareas de producción de atributos; (b) capturar la variedad de información de las entidades ontológicas (animales, artefactos, entre otros); (c) considerar la correspondencia con las regiones cerebrales de modalidad específica; (d) reflejar los canales establecidos de información sensorial; (e) reflejar experiencia introspectiva así como sensorio-motora (Cree \& McRae, 2003). Cada uno de los tipos de atributos propuestos está claramente definido y ejemplificado, lo cual facilita enormemente la replicabilidad de la clasificación. Esta taxonomía, que ciertamente posee un mayor grado de detalle y complejidad, es la que se utilizará en la presente investigación.

\section{Limitaciones de la Taxonomía Dicotómica}

La dicotomía de tipos de atributos ha demostrado ser insuficiente para explicar la complejidad de las representaciones conceptuales de las distintas categorías semánticas (Cree \& McRae, 2003; Marques, 2005; McRae \& Cree, 2002; Moss et al., 2007; Peraita, 2006). Particularmente, McRae y Cree (2002) han demostrado que los dominios semánticos se diferencian no sólo en los atributos sensoriales y funcionales sino en muchos otros tipos de atributos y que una 
taxonomía más compleja, como la propuesta por Wu y Barsalou (2009), puede reflejar mejor las diferencias entre los dominios ya que da cuenta más fielmente de la multidimensionalidad de la representación conceptual.

Por su parte, en habla hispana el grupo dirigido por Heminia Peraita ha propuesto un modelo de memoria semántica que plantea una clasificación de los atributos semánticos mucho más rica que el modelo sensorio-funcional. Este modelo acentúa la multidimensionalidad de la representación categorial. Es decir que la información que un sujeto tiene sobre los conceptos es rica y compleja. No solo incluye información de múltiples modalidades sino también diversos tipos de conocimientos abstractos. Los componentes conceptuales que estos autores proponen son: taxonómico, tipos, evaluativo/perceptual, funcional, parte-todo, procedimental, lugar/hábitat, actividad comportamental, ciclo vital y produce/genera (Peraita, Elosua, \& Linares, 1992).

\section{Insuficiencia de la Mera Frecuencia de Tipos de Atributos para Discriminar entre Dominios}

Modelos más recientes sobre la organización del conocimiento conceptual también ponen particular énfasis en la composición de atributos de las representaciones semánticas. Por ejemplo, el Enfoque de Estructura Conceptual considera que los conceptos pueden ser definidos en términos de los atributos que componen su significado. Sin embargo, a la hora de explicar la representación diferencial de las categorías y dominios semánticos, estos autores no ponen el acento sólo en el tipo de atributo sino que consideran que hay diversas cualidades de los mismos que determinan cómo el concepto es activado durante la comprensión y producción del lenguaje, y la manera en que es afectado por un daño (Moss, Tyler, \& Taylor, 2007). De este modo, afirman que la estructura de las representaciones semántica varía en los distintos dominios y categorías en función de la cantidad, cualidad e interacción entre los atributos semánticos que los componen.

Hay una serie de líneas de investigación recientes que se han propuesto estudiar cuáles son las cualidades que hacen a un atributo más central para la definición de un concepto (Sartori \& Lombardi, 2004; Sartori, Polezzi, Mameli, \& Lombardi, 2005; Montefinese, Ambrosini, Fairfield, \& Mammarella, 2014), o dicho en otros términos, qué variables determinan la contribución del atributo al núcleo del significado del concepto. Así, se han propuesto las variables Distintividad, Dominancia, Relevancia y Significancia para caracterizar a los atributos.

Se entiende por Distintividad al grado en que un atributo es compartido o no por varios conceptos (Devlin, Gonnerman, Andersen, \& Seidenberg, 1998; Garrard et al., 2001), es decir, es la medida en que una característica resulta específica de un cierto concepto. Por ejemplo, "tiene boca" es un atributo compartido por muchos animales, en cambio "tiene trompa" es un atributo de elefante apenas compartido con otros conceptos. De acuerdo con Cree, McNorgan y McRae (2006) los atributos distintivos tienen un estatus privilegiado en la organización de la memoria semántica ya que permiten diferenciar conceptos semejantes entre sí.

La Dominancia es una medida de la frecuencia con la cual un rasgo semántico es producido para definir a un concepto (Ashcraft, 1978). No se han reportado resultados que indiquen que esta medida por si solo contribuya en gran medida a explicar las diferencias en el procesamiento semántico de los dominios.

Por su parte, Sartori y Lombardi (2004) han propuesto la variable de Relevancia que conjuga a la Distintividad y la Dominancia. La misma es definida como una medida de la contribución de un atributo al núcleo del significado de un concepto (Sartori, Lombardi, \& Mattiuzzi, 2005). Por ejemplo, "tiene cuello largo" es un atributo muy relevante para jirafa, porque la mayoría de los sujetos utilizarían este atributo para definirlo, mientras que muy pocos utilizarían este atributo para definir otro concepto. Los investigadores afirman que mientras que pocos atributos de alta relevancia son suficientes para la recuperación precisa de un concepto, cuando la relevancia es baja la recuperación es inexacta (Sartori \& Lombardi, 2004; Sartori, et al., 2005). 
Más recientemente Montefinese, Ambrosini, Fairfield y Mammarella (2014) propusieron una variable denominada Accesibilidad que sería una medida que incluye tanto la frecuencia (o Dominancia) como el orden de producción del atributo en las Normas de Producción de Atributos Semánticos. Esta medida sumada a la Distintividad daría lugar a la Significancia. Estos autores demostraron su valor predictivo mediante tareas de verificación de atributos. En este mismo sentido Vivas, Lizarralde, Huapaya, Vivas y Comesaña (2014) desarrollaron recientemente un algoritmo informatizado denominado Definition Finder que permite procesar los atributos semánticos realizando un cálculo similar a la Accesibilidad y que permite unificar estos dos valores (frecuencia y orden de producción) en un único valor por atributo que va de 0 a 1 . Este cálculo se detalla en la metodología y será el que se utilizará en el presente trabajo para el análisis del peso de los tipos de atributos.

Utilizando las variables de los atributos hasta aquí mencionadas, trabajos realizados por Marques, Cappa y Sartori (2011) y Sartori, Polezzi, Mameli y Lombardi (2005) muestran que el tipo de atributos (sensorial vs. no sensorial) juegan un rol secundario en las tareas de nominación de la descripción con respecto a la Relevancia semántica. Similares resultados se encuentran en el trabajo de Marques (2005) analizando la Distintividad de los atributos. Este estudio indica que independientemente del tipo de atributo, los atributos distintivos fueron más seleccionados que los atributos compartidos en tareas de nominación tras la descripción.

Por su parte, un trabajo de Zannino, Perri, Pasqualetti, Caltaginore y Carlesimo (2006) comparó la Dominancia de los atributos a través de los dominios de acuerdo con los diferentes tipos de atributos. La misma fue establecida a partir de Normas de Producción de Atributos Semánticos. Estos autores arribaron al resultado de que la ventaja de los atributos sensoriales en el dominio de los seres vivos y funcionales para los objetos inanimados es observada sólo cuando se tiene en cuenta la Dominancia de los atributos; e iguales resultados se observan cuando se tiene en cuenta la Distintividad de los atributos.

\section{Utilización de Taxonomías a partir de Normas de Producción de Atributos Semánticos}

La colección de Normas de Producción de Atributos Semánticos ha sido un método frecuentemente utilizado para obtener datos referidos a los tipos de atributos que conforman la representación de los conceptos de manera empírica, como así también, para establecer la contribución de los atributos al núcleo del significado de los conceptos. La recolección de las Normas consiste en solicitar a un conjunto de hablantes de una lengua que emitan los atributos que a su entender mejor definen a un conjunto de conceptos. De allí se puede obtener numerosa información para conocer los tipos de atributos semánticos que caracterizan a cada dominio así como realizar análisis que permitan calcular variables fundamentales de los atributos como la Distintividad, Dominancia, Relevancia y Significatividad.

A partir de las Normas publicadas en idioma inglés, Cree y McRae (2003) han estudiado la tipología de atributos que caracteriza a cada dominio semántico basándose en la taxonomía propuesta por $\mathrm{Wu}$ y Barsalou (2009) descripta más arriba. A partir de los datos obtenidos mediante sus Normas, Cree y McRae calcularon la razón entre la cantidad de atributos de cada tipo para uno y otro dominio. El cálculo realizado se basó en la cantidad de atributos de cada tipo que apareció como definidor para cada dominio en las Normas. Sin embargo, al realizar este cálculo hay dos variables que no se contemplaron: la frecuencia de producción (es decir, la Dominancia) y el orden de producción de los atributos. Por un lado, la frecuencia con que se emite cada atributo es variable y brinda valiosa información acerca de la importancia que tiene el atributo para la definición del concepto. No es lo mismo un atributo que fue producido por 5 sujetos que otro que fue producido por 30. Por otro lado, al solicitar la producción de atributos de manera seriada también es importante contemplar el orden en que se emitió cada atributo. Si un atributo fue emitido con una frecuencia alta pero, a su vez, entre los primeros lugares es de esperar que 
su contribución al núcleo de significado del concepto sea mayor. En esta línea, la variable Accesibilidad permite conjugar Dominancia y orden de producción.

Este trabajo busca contribuir a describir las diferencias en la composición de atributos semánticos entre los dominios de seres vivos y objetos inanimados, partiendo de información obtenida mediante Normas de Producción de Atributos Semánticos en Español (Vivas et al., 2016) utilizando una taxonomía compleja y fundamentada y contemplando la Accesibilidad de tipos de atributos.

\section{Método}

\section{Participantes}

La muestra estuvo conformada por 180 estudiantes (122 mujeres y 58 varones) universitarios hispanoparlantes argentinos con edades comprendidas entre los 20 y 35 años $(M=23.62$ años; $S D=5.75$ ) que participaron en forma voluntaria bajo consentimiento informado. Se siguieron los principios de la Declaración de Helsinki (2013).

\section{Diseño}

Se seleccionaron 90 de los 400 conceptos de las Normas, cuyos referentes pictóricos, a su vez, se encuentran incluidos dentro del set de imágenes utilizadas por Cycowicz, Friedman, Rothstein y Snoodgrass (1997); 60 de los conceptos elegidos pertenecían a la categoría de seres vivos (30 correspondientes a animales y 30 a vegetales) y las 30 restantes a objetos inanimados (entre ellos, instrumentos musicales, medios de transporte y prendas de vestir). Estos conceptos fueron equiparados para ambos dominios semánticos teniendo en cuenta principalmente dos variables: la edad de adquisición y la familiaridad, obtenidas de normas Argentinas (Manoiloff, Artstein, Canavoso, Fernández, \& Segui, 2010).

\section{Procedimiento}

Los datos fueron recolectados de las Normas de Producción de Atributos Semánticos en español (Vivas, Vivas, Comesaña, García Coni,
\& Vorano, 2016). Para su construcción se les solicitó a los participantes que produjeran los atributos que mejor caractericen y describan a determinados conceptos. Luego de un minucioso proceso de unificación de las enunciaciones (para mayor detalle véase Vivas et al., 2016) se cargaron los descriptores en el programa Definition Finder (Vivas et al., 2014). Este programa, permite obtener un listado de atributos ponderados correspondientes a cada uno de los conceptos según su frecuencia y la ubicación en la que fueron emitidos dentro de todos los atributos brindados por un conjunto de participantes. De este proceso, se obtiene un valor particular de vinculación de cada atributo respecto al concepto dados en valores entre 0 y 1 (los pesos relativos fueron normalizados entre 0 y 1 para permitir la comparación entre conceptos). Así, por ejemplo, si para el concepto 'perro' muchas personas dijeron el atributo 'animal' y lo hicieron en primer lugar, este atributo tendrá un valor cercano a 1 , mientras que si pocas personas dijeron 'guardián' y lo hicieron en el cuarto o quinto lugar este atributo tendrá un valor cercano a 0 . A su vez, estos atributos fueron codificados de acuerdo al esquema de codificación propuesto por Wu y Barsalou (2009). Se escogió este esquema porque es el más completo de los publicados hasta el momento.

\section{Resultados}

El número medio de atributos dado por participante y por concepto en las Normas fue de 5.07. En primer lugar, se presentan los datos descriptivos de los tipos de atributos que fueron utilizados en las producciones de los participantes de las Normas ilustrados en las Figuras 1 y 2. Allí se observan las ponderaciones de los tipos de atributos generados para cada dominio de acuerdo al valor obtenido mediante su carga en el Definition Finder. Los atributos son presentados en orden decreciente en función de la ponderación promedio que obtuvieron para los conceptos del dominio correspondiente.

Se observa que el dominio de seres vivos está principalmente definido mediante atributos referidos a la categoría supraordinada (C-super), seguido por las propiedades de la superficie 


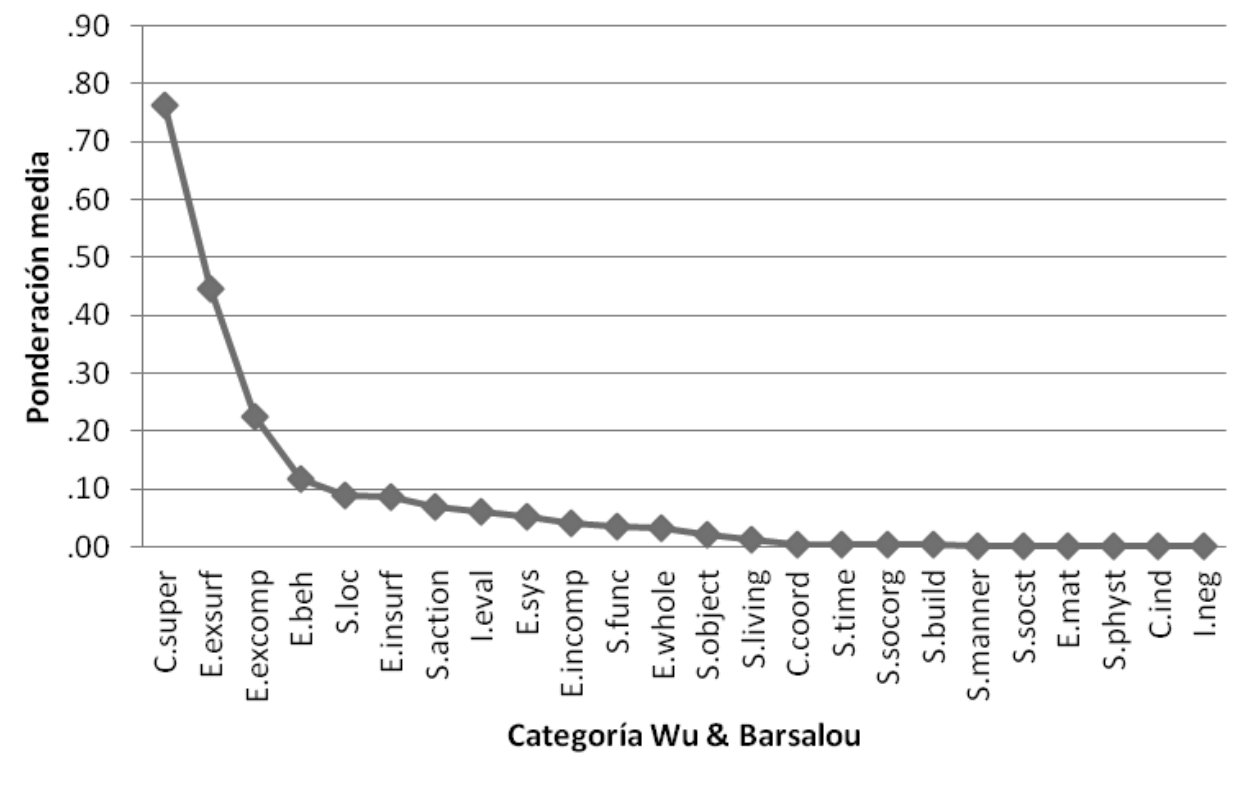

Figura 1. Descriptivos de atributos ponderados para el dominio de seres vivos.

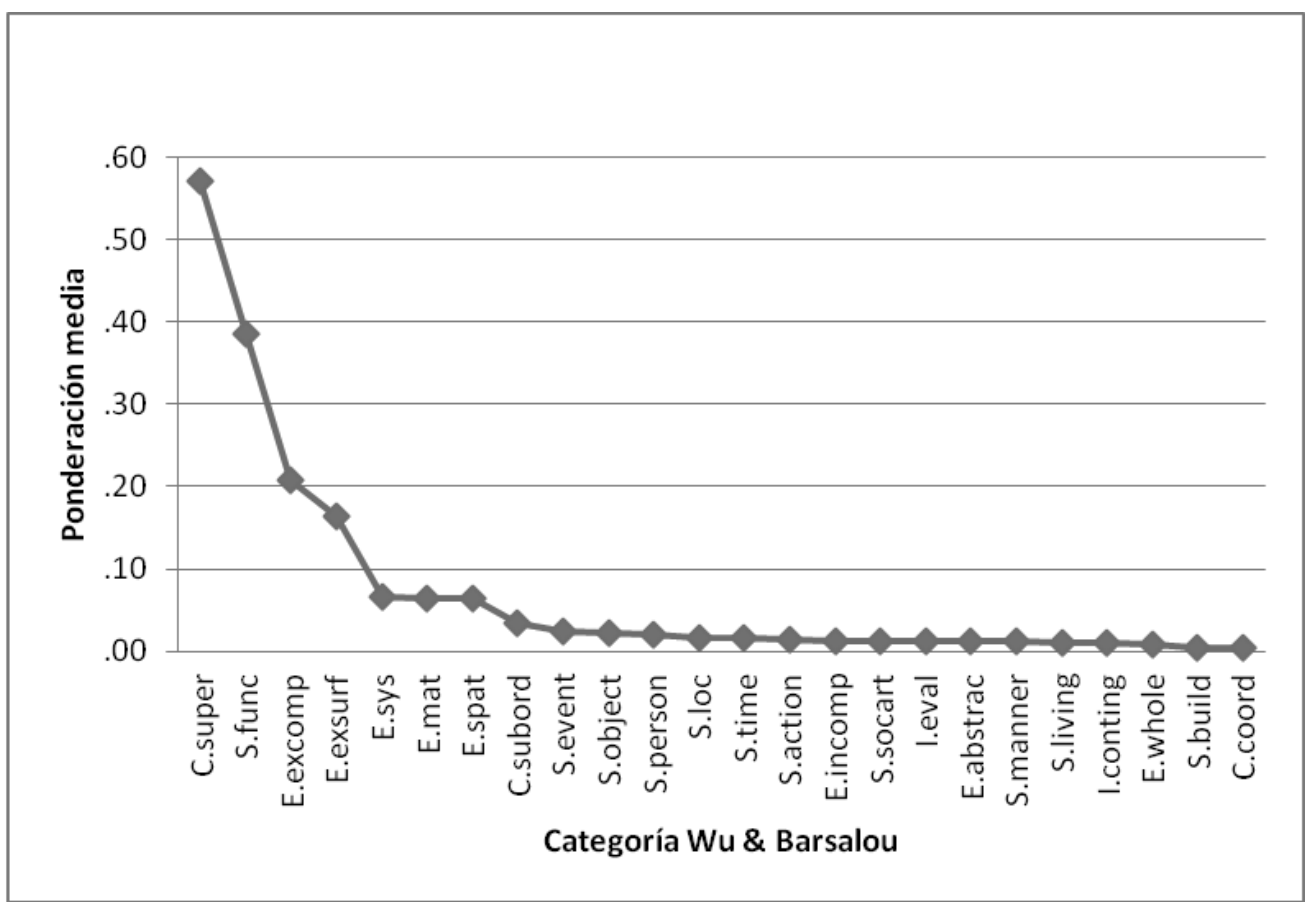

Figura 2. Descriptivos de atributos ponderados para el dominio de objetos inanimados

externa (E-exsurf), los componentes externos (E-excomp) y el comportamiento de la entidad (E-beh). Los demás tipos de atributos aparecen con puntuaciones por debajo del 0.1 , es decir, que represetan menos del $10 \%$ del peso total de los atributos definidores para ese concepto.

Por su parte, para los objetos inanimados se observa una mayor ponderación de los atributos referidos a la categoría supraordinada (C-super), seguidos por la función(S-fun), luego por los componentes externos (E-excomp) y por propiedades de la superficie externa (E-exsurf). En la Figura 3 se puede observar la secuencia de producción de los atributos de mayor peso para cada dominio.

En segundo lugar, se realizó una prueba $\mathrm{T}$ de diferencia de medias para comparar los valo- 
res de ponderación de cada tipo de atributo entre los dos dominios. Para realizar este cálculo, en aquellos conceptos para los cuales hubiera más de un atributo de cierto tipo (por ejemplo, tres E-excomp: TIENE_PATAS, TIENE_OREJAS y TIENE_TROMPA) se procedió a sumar la ponderación obtenida por cada atributo para el cálculo del valor del tipo de atributo (en el ejemplo E-excomp) para ese concepto. Los resultados indicaron una mayor ponderación de atributos $\mathrm{C}$ super $(\mathrm{T}=3.035 ; p=.003), \mathrm{S}-\operatorname{loc}(\mathrm{T}=3.343 ; p=$
$.001)$, S-action ( $\mathrm{T}=3.490 ; p=.001)$, E-incomp $(\mathrm{T}=1.988 ; p=.05) ; \mathrm{E}$-exsurf $(\mathrm{T}=5.326 ; p=$ $.001)$, E-insurf $(\mathrm{T}=3.805 ; p=.001), \mathrm{E}$-whole $(\mathrm{T}$ $=2.244 ; p=.027)$, E-beh $(\mathrm{T}=4.586 ; p=.001)$ e I-eval $(\mathrm{T}=2.998 ; p=.004)$ para el dominio de seres vivos y una mayor ponderación de C-subord $(\mathrm{T}=-2.135 ; p=.041)$, $\mathrm{S}$-func $(\mathrm{T}=-7.059 ; p$ $=.001)$, E-spat $(\mathrm{T}=-2.520 ; p=.017)$ y E-mat $(\mathrm{T}$ $=-3.819 ; p=.001)$ para los objetos inanimados. En la Figura 4 pueden observarse los atributos en los cuales se encontraron diferencias estadísticamente significativas entre los dominios.

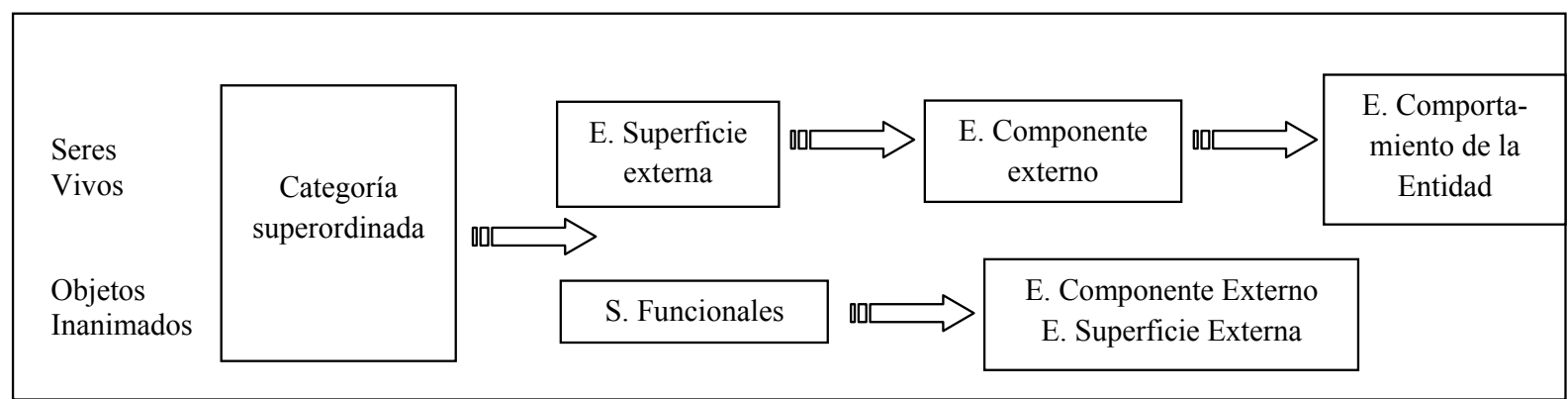

Figura 3. Orden de producción de atributos principales por dominio.

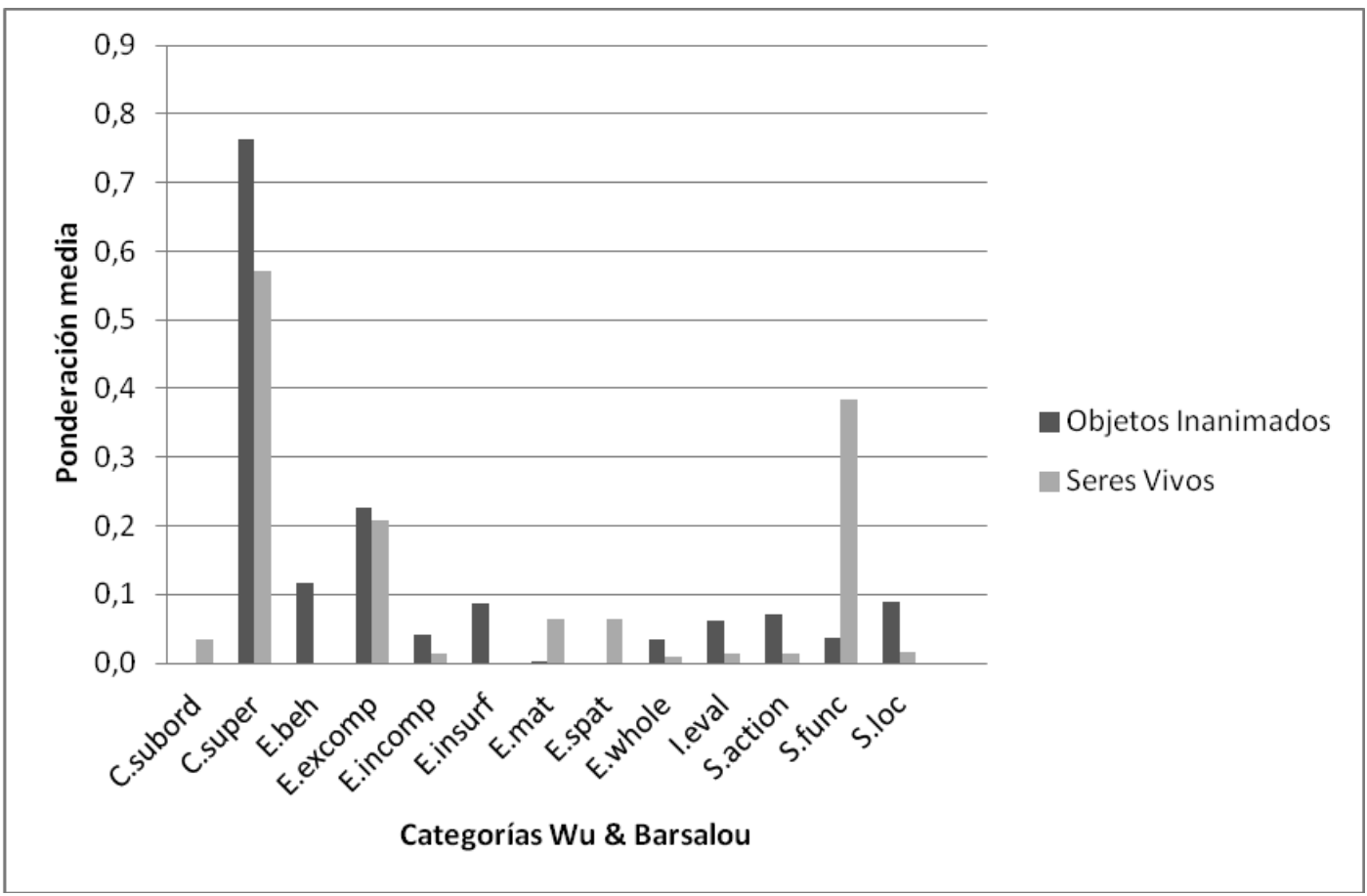

Figura 4. Diferencias entre dominios en la ponderación de los tipos de atributos.

\section{Discusión}

Como se mencionó al comienzo del artículo este trabajo se propuso describir los tipos de atributos que definen a los dominios semánticos buscando superar algunas de las críticas que se han realizado a los modelos sensorio-funcionales. La primera de dichas críticas está vinculada a la 
definición de los tipos de atributos. Las categorías "sensorial" y "funcional" no están explícitamente definidas y difieren entre distintos trabajos. Para salvar este escollo en la presente investigación se utilizó una taxonomía con definiciones claras y precisas sobre la clasificación utilizada. Como se especificó al inicio del artículo la taxonomía de tipos de atributos utilizada fue extraída de manera empírica a partir de la producción de atributos definidores de un conjunto de personas. Cabe suponer que este tipo de clasificación refleja más fielmente las cualidades de los atributos que una clasificación establecida a priori por el investigador. A su vez, cada categoría está claramente descripta y ejemplificada lo cual facilita su utilización por cualquier investigador que la quiera aplicar.

La segunda crítica apunta a la clasificación dicotómica de los tipos de atributos. Numerosos estudios demostraron que no sólo es insuficiente para diferenciar entre dominios (Cree \& McRae, 2003; McRae \& Cree, 2002; Moss et al., 2007; Peraita, 2006) sino que, en algunas ocasiones, tampoco se verifica la preponderancia entre atributos visuales para el dominio de seres vivos y funcionales para los objetos inanimados (Garrard et al., 2001; Vanoverberghe \& Storms, 2003). La taxonomía aquí utilizada cuenta con cinco grandes categorías y 44 tipos de atributos fundamentados empírica y teóricamente lo cual permite una mayor riqueza en la descripción de los tipos de atributos. Su conformación se basó en datos obtenidos de tareas de producción de atributos semánticos y las categorías se elaboraron en función del modelo de Conceptualización Situada (Barsalou, 2005). De este modo, esta taxonomía permite no sólo describir de manera más fina la composición de tipos de atributo por dominio sino también inferir el procesamiento cognitivo subyacente a la producción de los distintos tipos de atributos.

El tercer punto crítico, tiene que ver con la forma en que se mide el peso del atributo para cada concepto. Una posibilidad consiste simplemente en comparar la cantidad de atributos de cada tipo que definen a cada dominio semántico. En los trabajos más recientes los valores sobre los que se calculan las diferencias por dominio se extraen de Normas de Producción de Atributos Semánticos. Estas normas brindan información adicional, permitiendo calcular no sólo la frecuencia de producción de un atributo para determinado concepto, sino también realizar cálculos sobre la importancia de ese atributo para la definición del concepto en cuestión. De este modo, es posible analizar la contribución de los distintos tipos de atributos considerando además su peso ponderado para ese concepto. Como se mencionó anteriormente existen distintas formas de medir la contribución del atributo al núcleo de significado del concepto (Distintividad, Dominancia, Relevancia, Accesibilidad, Significancia). En el presente trabajo se calculó la Accesibilidad y se analizaron las diferencias entre dominios en función de este valor. Los trabajos realizados recientemente por Montefinesse et al. (2014) dan cuenta del poder explicativo de esta variable en tareas de verificación de atributos y de su importancia para capturar la saliencia de los atributos en la computación del significado de los conceptos. El presente trabajo también contribuye a demostrar su validez para ponderar los atributos definidores de los conceptos.

Teniendo en cuenta la superación de estos tres puntos críticos analizaremos los resultados obtenidos. En primer lugar, cabe resaltar algunas particularidades observadas en la descripción de los tipos de atributos que caracterizan a los dominios semánticos. Los resultados obtenidos aquí, indican que hay una preponderancia de los atributos de categoría supraordinada para ambos dominios. Es decir que el tipo de atributo más frecuente y que suele mencionarse en primer lugar es la categoría semántica de pertenencia del concepto. En este sentido, es importante resaltar que la población de la cual se seleccionó la muestra está conformada por estudiantes universitarios. Numerosos trabajos indican que los adultos jóvenes con alto nivel educativo tienen una tendencia a establecer categorizaciones taxonómicas por sobre otro tipo de relaciones (Murphy, 2001; Whitmore, Shore, \& Smith, 2004). Con lo cual, sería esperable que tomen como primer criterio de clasificación de los conceptos la categoría semántica.

Se observa en segundo lugar, un predominio de atributos referidos a propiedades sensoriales, particularmente visuales, según la clasificación 
de Wu y Barsalou (2009) propiedades de superficie externa y componente externo, para el dominio de seres vivos y de funcionales para los objetos inanimados tal como proponen los modelos sensorio-funcionales. En función de estos datos se puede elaborar una hipótesis acerca de los procesos cognitivos subyacentes, según el modelo de Conceptualización Situada y suponer que para ambos dominios se ingresaría primero a un nivel más abstracto que implica la delimitación de la categoría semántica y, posteriormente, para el dominio de seres vivos se activaría el simulador correspondiente al concepto individual y luego su contexto, mientras que para el dominio de objetos inanimados se recurriría primero a la conceptualización situada y luego al simulador específico del objeto.

En tercer lugar, aparece Comportamiento de la Entidad para seres vivos y propiedades sensoriales (de superficie externa y componente externo) para los objetos inanimados. Cabe mencionar que los seres vivos (particularmente los animales) no suelen tener estrictamente una función, pero su representación no es estática sino que incluye un aspecto dinámico ligado a un comportamiento. En este sentido, los atributos funcionales y el comportamiento de la entidad tienen en común el hecho de que remiten a pensar al objeto en acción. De acuerdo con el modelo de conceptualización situada propuesto por Barsalou (2005), cuando reactivamos los atributos referidos a un concepto estamos simulando parcialmente los encuentros que hemos tenido con ese objeto. Esta conceptualización no sólo incluye una imagen estática del aspecto del objeto sino también las acciones asociadas, las emociones y pensamientos referidos y los elementos que forman parte del contexto. A su vez, otros trabajos han considerado a todos aquellos atributos que describen una actividad o acción del objeto (animado o no) como parte de los atributos funcionales (Garrard et al., 2001).

Estos resultados, arrojan información relevante acerca del proceso cognitivo subyacente a la tarea de producción de atributos semánticos. Cuando se busca definir a un concepto lo primero que ocurriría es la delimitación de la categoría semántica de pertenencia, luego aparecerían los atributos más significativos para cada dominio: sensoriales-visuales para el dominio de seres vivos y funcionales para los objetos inanimados, en coincidencia con lo propuesto por los modelos sensorio-funcionales. Finalmente, aparecería un patrón inverso: aspectos dinámicos de los seres vivos (comportamiento de la entidad) y visuales de los objetos inanimados.

Hasta aquí se han comentado los resultados referidos a la descripción del flujo de producción de atributos para cada dominio. Sin embargo, otro de los objetivos del presente trabajo fue comparar los tipos de atributos entre dominios. Los datos obtenidos coinciden parcialmente con los de McRae y Cree (2002), quienes realizaron un estudio similar y observaron una preponderancia de atributos supraordinados, de superficie interna y comportamiento de la entidad para los seres vivos y subordinados, funcionales y material (o hecho de) para los objetos inanimados. En línea con esto, hay trabajos que indican que las relaciones taxonómicas (categoría supraordinada) serían más salientes para los objetos naturales y las temáticas (que implican conocimiento sobre elementos contextuales) para los artificiales (Kalènine et al., 2009). Los resultados obtenidos permitirían comprender este fenómeno en la medida en que los seres vivos se definen en mayor medida por su pertenencia categorial y sus cualidades físicas (atributos que se encuentran dentro de Propiedades de la Entidad) y los objetos inanimados por su función (atributo incluido por Wu y Barsalou dentro de las Propiedades Situacionales en la medida en que el objeto debe ser utilizado por alguien, lo cual sería una relación temática objeto-agente).

A pesar de estas similitudes, en el presente estudio también se observó un predominio de atributos de localización, acción, componente interno, superficie externa, parte-todo y evaluativo para los seres vivos y de atributos espaciales para los objetos inanimados. Cabe destacar que dentro de los atributos que presentan diferencias significativas, varios de ellos no tienen un peso sustancial en la composición de las representaciones conceptuales de los dominios. Por lo tanto, solamente los atributos supraordinados, superficie externa, funcionales y comportamiento de la entidad son los que cumplen con ambas condiciones. Esto indica que si bien la compo- 
sición de atributos semánticos de los conceptos es sumamente rica, hay ciertos atributos que tienen un peso fundamental y son los que definen y diferencian a los distintos dominios. Como se mencionó anteriormente, la Accesibilidad es una de las variables que permitiría medir ese peso.

En trabajos futuros, sería deseable poder conjugar los valores de Accesibilidad con la Distintividad para obtener la Significancia de los atributos utilizados y demostrar la eficacia predictiva de esta variable en tareas que tengan requerimientos de procesamiento inversos a la producción de atributos como verificación de atributos o reconocimiento de conceptos a partir de la presentación de atributos definidores. Este tipo de tareas permitiría medir si aumenta la eficacia en la respuesta en función de la Significancia de los atributos y contribuiría al conocimiento acerca de las variables que influyen en la composición de atributos de la representación conceptual.

\section{Referencias}

Ashcraft, M. H. (1978). Property norms for typical and atypical items from 17 categories: A description and discussion. Memory and Cognition, 6, 227-232. doi:10.3758/BF03197450

Barsalou, L. (2005). Situated conceptualization. In H. Cohen \& C. Lefebvre (Eds.), Handbook of Categorization in Cognitive Science (pp. 619650). Elsevier.

Capitani, E., Laiacona, M., Mahon, B., \& Caramazza, A. (2003). What are the facts of semantic category-specific deficits? A critical review of the clinical evidence. Cognitive Neuropsychology, 20(36), 213-261. doi:10.1080/02643290244000266

Caramazza, A., \& Shelton, J. R. (1998). Domainspecific knowledge systems in the brain: The animate-inanimate distinction. Journal of Cognitive Neuroscience, 10, 1-34. doi:10.1162/089892998563752

Cree, G. S., McNorgan, C., \& McRae, K. (2006). Distinctive features hold a privileged status in the computation of word meaning: Implications for theories of semantic memory. Journal of Experimental Psychology: Learning, Memory \& Cognition, 32, 643-658. doi:10.1037/02787393.32.4.643

Cree, G. S., \& McRae, K. (2003). Analyzing the factors underlying the structure and computation of the meaning of chipmunk, cherry, chisel, cheese and cello (and many other such concrete nouns). Journal of Experimental Psychology: General, 132(2), 163-201. doi:10.1037/00963445.132.2.163

Cycowicz, Y. M., Friedman D., Rothstein M., \& Snoodgrass, J. G. (1997). Picture naming by young children: Norms for name agreement, familiarity, and visual complexity. Journal of Experimental Child Psychology, 65, 171-237. doi:10.1006/jecp.1996.2356

Devlin, J. T., Gonnerman, L. M., Andersen, E. S., \& Seidenberg, M. S. (1998). Category-specific semantic deficits in focal and widespread brain damage: A computational account. Journal of Cognitive Neuroscience, 10, 77-94. doi:10.1162/089892998563798

Fargier, R., Ploux, S., Cheylus, A., Reboul, A., Paulignan, Y., \& Nazir T. A. (2014) Differentiating semantic categories during the acquisition of novel words: Correspondence analysis applied to event-related potentials. Journal of Cognitive Neuroscience, 26(11), 2552-2563. doi:10.1162/ jocn_a_00669

Farah, M. J., \& McClelland, J. L. (1991). A computational model of semantic memory impairment: Modality specificity and emergent category specificity. Journal of Experimental Psychology General, 120, 339-357. doi:10.1037/ 0096-3445.120.4.339

Garrard, P., Lambon Ralph, M. A., Hodges, M. A., \& Patterson, K. (2001). Prototypicality, distinctiveness, and intercorrelation: Analyses of the semantic attributes of living and nonliving concepts. Cognitive Neuropsychology, 18(2), 125-174. doi:10.1080/02643290125857

Johnson, M. K., Hermann, A. M., \& Bonilla, J. L. (1995). Semantic relations and Alzheimer's disease: Typicality and direction of testing. Neuropsychology, 9(4), 529-536. doi:10.1017/ S1355617700000709

Kalènine, S., Peyrin, S., Pichat, C., Segebarth, C., Bonthoux, F., \& Baciu, M. (2009). The sensory-motor specificity of taxonomic and thematic conceptual relations: A behavioral and fMRI study. Neuroimage, 44, 1152-1162. doi:10.1016/j.neuroimage.2008.09.043

Manoiloff, L., Artstein, M., Canavoso, M., Fernández, L., \& Segui, J. (2010). Expanded norms for 400 experimental pictures in an Argentinean Spanish speaking population. Behavior Research Methods, 42(2), 452-460. doi:10.3758/BRM.42.2.452 
Marques, J. F. (2005) Naming from definition: the role of feature type and feature distinctiveness. The Quarterly Journal of Experimental Psychology, 58A(4), 603-611. doi:10.1080/02724980443000106

Marques, J. F., Cappa, S. F., \& Sartori, G. (2011) Naming from definition, semantic relevance and feature type: The effects of aging and Alzheimer's disease. Neuropsychology, 25(1), 105-113. doi:10.1037/a0020417

Martínez-Cuitiño, M. (2007). Teorías del conocimiento conceptual. Revista Argentina de Neuropsicología, 9, 33-49. Recuperado en http:// www.revneuropsi.com.ar/images/stories/pdf/ martinezcuitinoranps9.pdf

McRae, K., \& Cree, G. S. (2002). Factors underlying category-specific semantic deficits. In E. M. E. Forde \& G. W. Humphreys (Eds.), CategorySpecificity in Brain and Mind (pp. 211-249). East Sussex, UK: Psychology Press.

Montefinese, M., Ambrosini, E., Fairfield, B., \& Mammarella, N. (2014). Semantic significance: A new measure of feature salience. Memory \& Cognition, 42(3), 355-369. doi:10.3758/s13421013-0365-y

Moss, H. E., Tyler, L. K., \& Taylor, K. I. (2007) Conceptual structure. In M. Gareth Gaskell (Ed.), The Oxford Handbook of Psycholinguistics. Oxford University Press.

Murphy, G. L. (2001). Causes of taxonomic sorting by adults: A test of the thematic-to-taxonomic shift. Psychonomic Bulletin \& Review, 8(4), 834-839. doi:10.3758/BF03196225

Peraita, H. (2006). ¿Es la dicotomía entre atributos sensorial-perceptivos y funcional-asociativos suficiente para explicar las disociaciones categoriales y el deterioro semántico? Una crítica a la hipótesis sensorio-funcional. In J. C. González (Ed.), Perspectivas contemporáneas sobre la cognición. Percepción, categorización, conceptualización (pp. 237-264). México: Siglo XXI.

Peraita, H., Elosua, R., \& Linares, P. (1992). Representación de categorías naturales en niños ciegos. Madrid: Editorial Trotta.

Sartori, G., \& Lombardi, L. (2004). Semantic relevance and semantic disorders. Journal of Cognitive Neuroscience, 16, 439-452. doi:10.1016/j. neuropsychologia.2006.08.028

Sartori, G., Lombardi, L., \& Mattiuzzi, L. (2005). Semantic relevance best predicts normal and abnormal name retrieval. Neuropsychologia, 43, 754-770. doi:10.1016/j.neuropsychologia.2004.08.001

Sartori, G., Polezzi, D., Mameli F., \& Lombardi, L. (2005). Feature type effects in semantic memory: An event related potentials study. Neuroscience Letters, 390, 139-144. doi:10.1016/j. neulet.2005.08.015

Vanoverberghe, V., \& Storms, G. (2003) Feature importance in feature generation and typicality rating. European Journal of Cognitive Psychology, 15(1), 1-18. doi:10.1080/09541440303600

Vivas, J., Vivas, L., Comesaña, A., García Coni. A., \& Vorano, A. (2016). Spanish semantic feature production norms for 400 concrete concepts. Behavior Research Methods, en prensa. doi 10.3758/s13428-016-0777-2

Vivas, J., Lizarralde, F., Huapaya, R., Vivas, L., \& Comesaña, A. (2014). Organización reticular de la memoria semántica. Natural Finder y Definition Finder, dos métodos informatizados para recuperar conocimiento. Encontros Bibli, 19(40), 235-252. doi:10.5007/15182924.2014v19n40p235

Warrington, E. K., \& Shallice, T. (1984) Category specific semantic impairments. Brain, 107, 829854. doi:10.1093/brain/107.3.829

Whitmore, J. M., Shore, W. J., \& Smith, P. H. (2004). Partial knowledge of Word Meanings: Thematic and taxonomic representations. Journal of Psycholinguistic Research, 33(2), 137-164. doi:10.1023/B:JOPR.0000017224.21951.0e

Wu, L. L., \& Barsalou, L. W. (2009). Perceptual simulation in conceptual combination: Evidence from property generation. Acta Psychologica, 132, 173-189. doi:10.1016/j.actpsy.2009.02.002

Zannino, G. D., Perri, R., Pasqualetti, P., Caltagirone, C., \& Carlesimo, G. A. (2006) Analysis of the semantic representations of living and nonliving concepts: A normative study. Cognitive Neuropsychology, 23(4), 515-540. doi:10.1080/02643290542000067 